\title{
Review: non-parental daycare promotes disadvantaged children's intelligence, development, and school achievement
}

\author{
Zoritch B, Roberts I, Oakley A. The health and welfare effects of day-care: a systematic review of randomised controlled trials. Soc \\ Sci Med 1998 Aug;47:317-27.
}

\section{Question}

What are the effects of non-parental daycare on educational and health and welfare outcomes for disadvantaged children and their families?

\section{Data sources}

Studies were identified by searching 7 electronic databases (Medline, EMBASE/Excerpta Medica, the Cochrane Controlled Trial Register, the Social Science Citation Index, PsycLIT, ERIC [Educational Resources Information Center], and BIRD [French language database]); handsearching 2 journals from 1977-96 (Child Development and Journal of Child Abuse and Neglect); reviewing the bibliographies of 1 relevant non-systematic review of daycare, relevant papers, books, trial reports and review articles, and conference proceedings; and contacting researchers in the field.

\section{Study selection}

Studies were selected if the intervention involved the provision of non-parental daycare of children $<5$ years of age and if the trial design was that of a randomised or quasi-randomised controlled trial.

\section{Data extraction}

Data were extracted on changes in child's developmental and intelligence quotient (IQ), school performance and attitude, and behaviour and health; and maternal employment, fertility, and interaction with children.

\section{Main results}

A total of 8 trials were selected after examining 920 abstracts or papers and 19 books. Because of heterogeneity of the interven tions a meta-analysis was not done. In total, 2203 children were randomised and follow up ranged from 6 months to 27 years. All trials were done in the US. 6 of the 8 trials specifically targeted disadvantaged families of lower socioeconomic status. All studies showed that IQ was increased by participation in daycare, but in most studies this beneficial effect disappeared 1 or 2 years after the intervention ended. However, the early cognitive gain was associated with later reduction in school failure. All but 1 of the studies that measured school achievement showed increased school achievement with daycare (ie, fewer children were placed in special education classes, fewer failed a grade, and a greater number showed better competence in reading, writing, mathematics, and general knowledge). The 3 studies that evaluated children's behaviour found that daycare led to improved classroom and personal behaviour, more academic motivation, and less delinquent and disruptive behaviour. Mother-child interactions appeared to be better with enriched daycare, and more mothers with children in daycare had a higher education and more stable employment. 1 study evaluated the long term effect of daycare (27 year follow up) and found that more children in the daycare group had jobs at 19 years of age, and fewer were on welfare, pregnant, or had engaged in criminal acts.

\section{Conclusion}

Early education programmes in the form of enriched nonparental, out of home, daycare promote disadvantaged children's intelligence, development, and school achievement.

Source of funding: National Health Services and the Institute of Education.

For correspondence:Dr B Zoritch, St. Peter's Hospital, Children's Unit, Guilford Road, Chertsey, Surrey KT16 OPZ, UK. Fax +44 (0)1932 875171 .

\section{Commentary}

This review by Zoritch et al focuses on studies evaluating early intervention programmes to enhance the skills of disadvantaged children rather than on daycare per se. It is disappointing to learn that so few studies met the criteria for this review. Even those reports included were noted to have methodological flaws. It is rare to find valid studies, particularly in the evaluation of complex services. Designing methodologically sound studies to evaluate early education programmes is a major challenge, one that is labour intensive and financially demanding. An important lesson from this review is that reliable answers to important social questions are hard to come by.

Methodological limitations lend caution to the findings but the outcomes were consistent. Each study entailed some benefits, but it would be a mistake to take away the message that all types of "out of home" care are good for young children. Programmes with specific educational aims, especially those subjected to research evaluation, are usually high quality exemplary programmes tied to academic centres. Few of the options available to most parents seeking care for their young children will match the characteristics of the programmes targeted in this review. Nevertheless, the success of such programmes shows that "out of home" care is not, in and of itself, deleterious and that well designed early education programmes offer potential benefits to both children and parents.

It is noteworthy that in most studies early gains were not maintained; some advantages emerged only in longer term follow up; and the project with the longest follow up (27 years) noted "real life" advantages (eg, fewer welfare cases and arrests; more jobs, marriages, and homeowners). This parallels the multicentre long term evaluation of a consortium of early intervention programmes in the US. ${ }^{1}$ Although early gains in IQ scores, school grades, and/or social skills were often lost several years later, long term gains were evident in adulthood. When intervening with disadvantaged youngsters, these adult outcomes are the real fulfilment of our hopes. Taken together, these findings and earlier work indicate that even if immediate gains are lacking, long term payoff can result. Susan Goldberg, $\mathrm{PhD}$ The Hospital for Sick Children Toronto, Ontario, Canada

1 Lazar I, Darlington RB, Murray H, et al. Lasting effects of early education. Monographs of the Society for Research in Child Development, no 47. 1982: serial no 195 . 